\title{
Patch contribution to near-field radiative energy transfer and van der Waals pressure between two half-spaces
}

\author{
Yi Zheng and Arvind Narayanaswamy* \\ Department of Mechanical Engineering, Columbia University, New York, New York 10027, USA
}

(Received 6 November 2013; published 18 February 2014)

\begin{abstract}
Near-field effects in fluctuational electrodynamics leads to enhancement of radiative energy transfer as well as the emergence of van der Waals and/or Casimir pressure. While much has been learned from the analysis of near-field interactions between two half-spaces separated by a vacuum gap, we shed new light on the problem by finding how much of a surface patch on one of the half-spaces contributes to the energy transfer or van der Waals pressure at any location within the vacuum gap. We show that energy transfer and fluctuation-induced van der Waals pressure at any point on the surface of one half-space are qualitatively and quantitatively different due to the dissimilar zones of influence of interactions. We also show that the contributions from different surface patches are qualitatively similar for half-spaces with dielectric materials (silica, silicon carbide) and half-spaces with metals (gold).
\end{abstract}

DOI: 10.1103/PhysRevA.89.022512

PACS number(s): 31.30.jh, 03.50.De, 42.50.Ct, 44.40.+a

\section{INTRODUCTION}

Energy and momentum transfer via electromagnetic waves due to zero-point and thermal fluctuations are best described by Rytov's theory of fluctuational electrodynamics [1,2]. Though of common origin, the two phenomena are different in one significant way: Finite thermal energy transfer is a thermal nonequilibrium phenomenon, whereas van der Waals pressure can exist irrespective of temperatures of the objects. In fact, it is the equilibrium contribution to pressure that dominates over thermal nonequilibrium contribution at small gaps [3]. Both energy and momentum transfer between parallel halfspaces separated by a vacuum gap have been studied by many researchers over the last seven decades [4-9]. The expressions for thermal radiative and momentum transfer between two half-spaces (as shown in Fig. 1) separated by a gap $d$ can be obtained as follows:

$$
\begin{aligned}
Q_{1 \rightarrow 2} & =\int_{0}^{\infty} \frac{d \omega}{2 \pi} \Theta\left(\omega, T_{1}\right) T_{1 \rightarrow 2}^{(e)}(\omega), \\
F_{1 \rightarrow 2} & =\int_{0}^{\infty} \frac{d \omega}{2 \pi} \Theta\left(\omega, T_{1}\right) T_{1 \rightarrow 2}^{(m)}(\omega),
\end{aligned}
$$

where $T_{1 \rightarrow 2}^{(e)}$ and $T_{1 \rightarrow 2}^{(m)}$ are the generalized transmissivities for energy [10,11] and momentum transfer [11]; $Q_{1 \rightarrow 2}$ and $F_{1 \rightarrow 2}$ are the energy flow rate per unit area and pressure (or momentum flow rate per unit area), respectively, on the surface of half-space 2 due to fluctuational sources within halfspace $1 ; \Theta\left(\omega, T_{1}\right)=(\hbar \omega / 2) \operatorname{coth}\left(\hbar \omega / 2 k_{B} T_{1}\right) .2 \pi \hbar$ is Planck's constant, $k_{B}$ is Boltzmann constant, and $T_{1}$ is the temperature of half-space 1. Expressions for $T_{1 \rightarrow 2}^{(e)}$ and $T_{1 \rightarrow 2}^{(m)}$ between two parallel half-spaces separated by a vacuum gap are given by $[10,11]$

$$
\begin{aligned}
T_{1 \rightarrow 2}^{(e)}(\omega)= & \sum_{\mu=s, p}\left[\int_{0}^{k_{0}} \frac{k_{\rho} d k_{\rho}}{2 \pi} \frac{\left(1-\left|\widetilde{R}_{v 1}^{(\mu)}\right|^{2}\right)\left(1-\left|\widetilde{R}_{v 2}^{(\mu)}\right|^{2}\right)}{\left|1-\widetilde{R}_{v 1}^{(\mu)} \widetilde{R}_{v 2}^{(\mu)} e^{i 2 k_{v v} d}\right|^{2}}\right. \\
& \left.+\int_{k_{0}}^{\infty} \frac{k_{\rho} d k_{\rho}}{2 \pi} \frac{4 \operatorname{Im}\left(\widetilde{R}_{v 1}^{(\mu)}\right) \operatorname{Im}\left(\widetilde{R}_{v 2}^{(\mu)}\right) e^{-2\left|k_{z v}\right| d}}{\left|1-\widetilde{R}_{v 1}^{(\mu)} \widetilde{R}_{v 2}^{(\mu)} e^{-2\left|k_{z v}\right| d}\right|^{2}}\right],
\end{aligned}
$$

\footnotetext{
*arvind.narayanaswamy@ columbia.edu
}

$$
\begin{aligned}
T_{1 \rightarrow 2}^{(m)}(\omega)= & \sum_{\mu=s, p}\left[-\int_{0}^{k_{0}} \frac{k_{\rho} d k_{\rho}}{2 \pi} \frac{k_{z v}}{\omega} \frac{\left(1-\left|\widetilde{R}_{v 1}^{(\mu)}\right|^{2}\right)\left(1+\left|\widetilde{R}_{v 2}^{(\mu)}\right|^{2}\right)}{\left|1-\widetilde{R}_{v 1}^{(\mu)} \widetilde{R}_{v 2}^{(\mu)} e^{i 2 k_{z v} d}\right|^{2}}\right. \\
& \left.+\int_{k_{0}}^{\infty} \frac{k_{\rho} d k_{\rho}}{2 \pi} \frac{\left|k_{z v}\right|}{\omega} \frac{4 \operatorname{Im}\left(\widetilde{R}_{v 1}^{(\mu)}\right) \operatorname{Re}\left(\widetilde{R}_{v 2}^{(\mu)}\right) e^{-2\left|k_{z v}\right| d}}{\left|1-\widetilde{R}_{v 1}^{(\mu)} \widetilde{R}_{v 2}^{(\mu)} e^{-2\left|k_{z v}\right| d}\right|^{2}}\right],
\end{aligned}
$$

where the integrals of the form $\int_{0}^{k_{0}} d k_{\rho} f\left(k_{\rho}\right)$ and $\int_{k_{0}}^{\infty} d k_{\rho} f\left(k_{\rho}\right)$ correspond to contributions due to propagating and evanescent waves, respectively, and the superscripts $e$ and $m$ stand for energy and momentum transfer, respectively. $k_{0}=\omega / c, k_{\rho}$ is the magnitude of the in-plane wave vector, $k_{z v}=\sqrt{k_{0}^{2}-k_{\rho}^{2}}$ is $z$-component wave vector in vacuum, $d$ is the vacuum gap, $\widetilde{R}_{v i}^{(\mu)}$ is the generalized Fresnel reflection coefficient [12,13] for $\mu$-polarized plane waves at the interface between vacuum and half-space $i=1,2$, and superscript $\mu=s, p$ stands for transverse electric and transverse magnetic polarizations, respectively.

In what way are we adding anything new to this vast amount of literature? First, we are not calculating only the energy flux or van der Waals pressure at any point in the vacuum gap. What we are calculating is the contribution to the energy flux or van der Waals pressure at any location on the surface of one half-space attributable to different portions of the surface of the other half-space. The key result of this work is encapsulated in Fig. 5. As we will show, the calculation is by no means a trivial extension of existing methods [3,14]. Second, for calculating van der Waals pressure, we could no longer use Lifshitz' elegant (and better suited for computations) method of evaluating Green's functions at imaginary frequencies (Matsubara frequencies) [15]. We have had to rely on computations along the real frequency axis $[14,16]$. Third, we show that different portions of a half-space contribute differently to energy flux and van der Waals pressure at any location in the vacuum gap.

The structure of this paper is as follows: In Sec. II, we use the fluctuational electrodynamics to express the electric and magnetic field correlation functions in terms of surface integrals of the dyadic Green's functions. In Sec. III, we show 


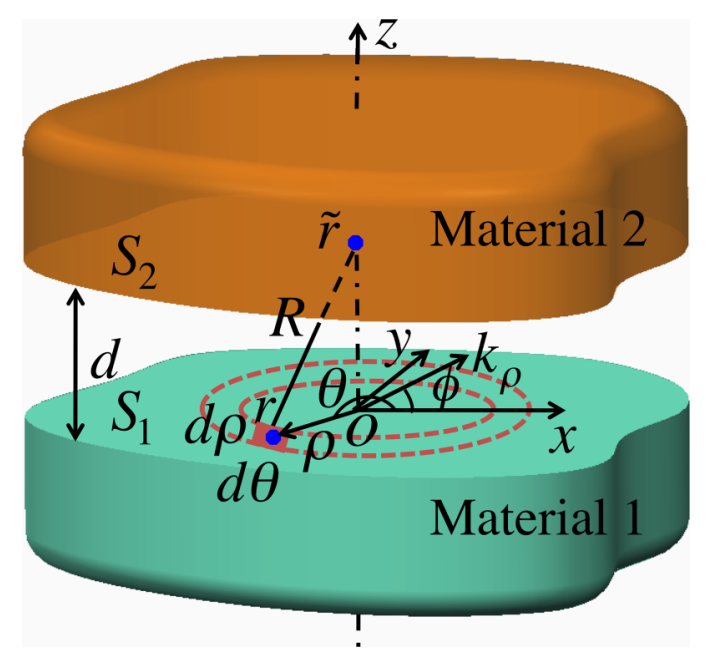

FIG. 1. (Color online) A schematic diagram of surface patch contribution between two half-spaces. The surfaces $S_{1}$ and $S_{2}$ correspond to the planes $z=0$ and $z=d$. $\tilde{\mathbf{r}}$ is the location with coordinate $(0,0, d)$. Radiative energy and momentum transfer are evaluated at vector $\tilde{\mathbf{r}}$ due to a surface patch of area $\rho d \rho d \theta$ containing the source vector $\mathbf{r}$ on $S_{1}$.

how the contribution to energy flux and van der Waals pressure at any point in the vacuum gap can be attributed to any patch on the surface. In Sec. III A, we show how to achieve the surface decomposition for energy flux and van der Waals pressure. In Sec. III B, we give details of the computations along the real frequency axis, especially important at high frequencies because of the oscillations that occur in the contribution to van der Waals pressure as a function of frequency as well as position of the surface patch relative to the point at which the van der Waals pressure is calculated. In Secs. III A-III B, we assume that the half-spaces are made of silica in order to elaborate our method. In Sec. IV, we calculate the partial contributions to radiative transfer and pressure from different portions of one half-space to any point in vacuum. We also show that the contributions from different surface patches are qualitatively similar for half-spaces with dielectric materials (silica, silicon carbide) and half-spaces with metals (gold). For all the materials in this work, optical data are taken from Palik [17]. We summarize our work in Sec. V.

\section{FLUCTUATIONAL ELECTRODYNAMICS AND GREEN'S FUNCTION FORMALISM}

Because our focus is on determining the energy transfer rate and pressure at any point on one of the half-spaces due to a part of the second half-space, we cannot use Eqs. (2) and (3) directly. Consider the electric and magnetic fields $\mathbf{E}$ and $\mathbf{H}$, respectively, due to thermal sources within an object $i(i=1,2$ for the configuration shown in Fig. 1). Since the electric and magnetic fields are assumed to be stationary, $\langle\mathbf{E}(\tilde{\mathbf{r}}, t) \mathbf{E}(\tilde{\mathbf{r}}, t)\rangle^{(i)}$, $\langle\mathbf{H}(\tilde{\mathbf{r}}, t) \mathbf{H}(\tilde{\mathbf{r}}, t)\rangle^{(i)}$, and $\langle\mathbf{E}(\tilde{\mathbf{r}}, t) \mathbf{H}(\tilde{\mathbf{r}}, t)\rangle^{(i)}$ are independent of time [18]. The superscript $(i)$ is used to indicate that the field correlation is due to fluctuations within object $i$. The equal time correlation functions are related to the cross-spectral densities by

$$
\begin{aligned}
\left\langle E_{p}(\tilde{\mathbf{r}}, t) E_{q}(\tilde{\mathbf{r}}, t)\right\rangle_{s}^{(i)} & =\int_{0}^{\infty} \frac{d \omega}{2 \pi}\left\langle E_{p}(\tilde{\mathbf{r}}) E_{q}^{*}(\tilde{\mathbf{r}})\right\rangle_{s}^{(i)}, \\
\left\langle H_{p}(\tilde{\mathbf{r}}, t) H_{q}(\tilde{\mathbf{r}}, t)\right\rangle_{s}^{(i)} & =\int_{0}^{\infty} \frac{d \omega}{2 \pi}\left\langle H_{p}(\tilde{\mathbf{r}}) H_{q}^{*}(\tilde{\mathbf{r}})\right\rangle_{s}^{(i)}, \\
\left\langle E_{p}(\tilde{\mathbf{r}}, t) H_{q}(\tilde{\mathbf{r}}, t)\right\rangle_{s}^{(i)} & =\int_{0}^{\infty} \frac{d \omega}{2 \pi}\left\langle E_{p}(\tilde{\mathbf{r}}) H_{q}^{*}(\tilde{\mathbf{r}})\right\rangle_{s}^{(i)},
\end{aligned}
$$

where the subscript $s$ in Eqs. (4a)-(4c) implies a symmetric sum. $\langle\cdot\rangle$ denotes the ensemble average. In Eqs. (4a)-(4c), though $\omega$ does not appear explicitly as an argument of $E_{p}(\tilde{\mathbf{r}})$ and $H_{p}(\tilde{\mathbf{r}})(p=1,2,3)$, they are $\omega$ dependent.

Using Rytov's theory of fluctuational electrodynamics $[19,20]$, the cross-spectral densities of the components of the electric and magnetic fields at $\tilde{\mathbf{r}}$ in vacuum due to the thermal sources within object $i(i=1,2)$, occupying volume $V_{i}$ and bounded by closed surface $S_{i}$ (if the objects are half-spaces, as shown in Fig. 1, the surfaces are not closed surfaces), are [11]

$$
\begin{aligned}
\left\langle\mathbf{E}(\tilde{\mathbf{r}}) \mathbf{E}^{*}(\tilde{\mathbf{r}})\right\rangle_{s}^{(i)} & =2 \omega \mu_{o} \Theta\left(\omega, T_{i}\right) \overline{\overline{\mathcal{E}}}^{(i)}(\tilde{\mathbf{r}}), \\
\left\langle\mathbf{H}(\tilde{\mathbf{r}}) \mathbf{H}^{*}(\tilde{\mathbf{r}})\right\rangle_{s}^{(i)} & =2 \omega \varepsilon_{o} \Theta\left(\omega, T_{i}\right) \overline{\overline{\mathcal{H}}}^{(i)}(\tilde{\mathbf{r}}), \\
\left\langle\mathbf{E}(\tilde{\mathbf{r}}) \mathbf{H}^{*}(\tilde{\mathbf{r}})\right\rangle_{s}^{(i)} & =\Theta\left(\omega, T_{i}\right) \overline{\overline{\mathcal{X}}}^{(i)}(\tilde{\mathbf{r}}),
\end{aligned}
$$

where $\overline{\overline{\mathcal{E}}}^{(i)}(\tilde{\mathbf{r}}), \overline{\overline{\mathcal{H}}}^{(i)}(\tilde{\mathbf{r}})$, and $\overline{\overline{\mathcal{X}}}^{(i)}(\tilde{\mathbf{r}})$ are given by

$$
\overline{\overline{\mathcal{E}}}^{(i)}(\tilde{\mathbf{r}})=2 \operatorname{Im} \oint_{S_{i}}\left[\mu(\mathbf{r}) \overline{\bar{G}}_{e}^{T}(\mathbf{r}, \tilde{\mathbf{r}})\right]^{*} \cdot\left[\mathbf{n}_{i}(\mathbf{r}) \times \overline{\bar{G}}_{E}(\mathbf{r}, \tilde{\mathbf{r}})\right] d s(\mathbf{r}),
$$

$$
\overline{\overline{\mathcal{H}}}^{(i)}(\tilde{\mathbf{r}})=2 \operatorname{Im} \oint_{S_{i}}\left[\varepsilon(\mathbf{r}) \overline{\bar{G}}_{m}^{T}(\mathbf{r}, \tilde{\mathbf{r}})\right]^{*} \cdot\left[\mathbf{n}_{i}(\mathbf{r}) \times \overline{\bar{G}}_{M}(\mathbf{r}, \tilde{\mathbf{r}})\right] d s(\mathbf{r}),
$$

$$
\begin{aligned}
\overline{\overline{\mathcal{X}}}^{(i)}(\tilde{\mathbf{r}})= & -\oint_{S_{i}}\left\{\overline{\bar{G}}_{E}^{T}(\mathbf{r}, \tilde{\mathbf{r}}) \cdot\left[\mathbf{n}_{i}(\mathbf{r}) \times \overline{\bar{G}}_{M}^{*}(\mathbf{r}, \tilde{\mathbf{r}})\right]+\frac{\omega^{2}}{c^{2}}\right. \\
& \left.\times \mu(\mathbf{r}) \overline{\bar{G}}_{e}^{T}(\mathbf{r}, \tilde{\mathbf{r}}) \cdot\left[\mathbf{n}_{i}(\mathbf{r}) \times \varepsilon(\mathbf{r}) \overline{\bar{G}}_{m}(\mathbf{r}, \tilde{\mathbf{r}})\right]^{*}\right\} d s(\mathbf{r}),
\end{aligned}
$$

where $\mathbf{n}_{i}(\mathbf{r})$ is the outward pointing unit normal vector at $\mathbf{r} \in$ $S_{i}, \overline{\bar{G}}_{e}(\mathbf{r}, \tilde{\mathbf{r}})$ and $\overline{\bar{G}}_{m}(\mathbf{r}, \tilde{\mathbf{r}})$ are electromagnetic dyadic Green's functions (DGFs) of the vector Helmholtz equation [21,22], and $\overline{\bar{G}}_{E}(\mathbf{r}, \tilde{\mathbf{r}})=\nabla \times \overline{\bar{G}}_{e}(\mathbf{r}, \tilde{\mathbf{r}}), \overline{\bar{G}}_{M}(\mathbf{r}, \tilde{\mathbf{r}})=\nabla \times \overline{\bar{G}}_{m}(\mathbf{r}, \tilde{\mathbf{r}})$. The $\nabla \times$ operation is done on the position vector $\mathbf{r}$. From Eq. (6c), the normal component of the Poynting vector at a location $\tilde{\mathbf{r}}$ on the surface of object 2 due to the thermal sources on the surface of object 1 is given by [11]

$$
Q_{1 \rightarrow 2}(\tilde{\mathbf{r}})=\int_{0}^{\infty} \frac{d \omega}{2 \pi} \Theta\left(\omega, T_{1}\right) \oint_{S_{1}} d s(\mathbf{r}) S_{1 \rightarrow 2}^{(e)}(\tilde{\mathbf{r}}, \mathbf{r}, \omega),
$$

where $S_{1 \rightarrow 2}^{(e)}(\tilde{\mathbf{r}}, \mathbf{r}, \omega)$ is given by

$$
\begin{aligned}
S_{1 \rightarrow 2}^{(e)}(\tilde{\mathbf{r}}, \mathbf{r}, \omega)= & 2 \operatorname{Re} \operatorname{Tr}\left[\frac{\omega^{2}}{c^{2}}\left[\mathbf{n}_{1}(\mathbf{r}) \times \mu(\mathbf{r}) \overline{\bar{G}}_{e}(\mathbf{r}, \tilde{\mathbf{r}})\right]\right. \\
& \cdot\left[\mathbf{n}_{2}(\tilde{\mathbf{r}}) \times \varepsilon(\tilde{\mathbf{r}}) \overline{\bar{G}}_{m}(\tilde{\mathbf{r}}, \mathbf{r})\right]^{*}+\left[\mathbf{n}_{1}(\mathbf{r}) \times \overline{\bar{G}}_{E}(\mathbf{r}, \tilde{\mathbf{r}})\right] \\
& \left.\cdot\left[\mathbf{n}_{2}(\tilde{\mathbf{r}}) \times \overline{\bar{G}}_{E}(\tilde{\mathbf{r}}, \mathbf{r})\right]^{*}\right] .
\end{aligned}
$$


In Eq. (8), the vectors $\mathbf{n}_{1}(\mathbf{r})$ and $\mathbf{n}_{2}(\tilde{\mathbf{r}})$ are outward pointing unit normal vectors at $\mathbf{r}$ and $\tilde{\mathbf{r}}$ on the surfaces of objects 1 and 2 , respectively. Re stands for "real part" and the operator $\mathrm{Tr}$ denotes "trace."

van der Waals pressure in vacuum can be determined from the Maxwell stress tensor [15,23], $\overline{\bar{\sigma}}=\overline{\bar{\sigma}}^{e}+\overline{\bar{\sigma}}^{m}$, where $\overline{\bar{\sigma}}^{e}$ and $\overline{\bar{\sigma}}^{m}$ are the electric and magnetic field contributions, respectively. $\overline{\bar{\sigma}}^{e}$ and $\overline{\bar{\sigma}}^{m}$ are given by

$$
\begin{aligned}
& \overline{\bar{\sigma}}^{e}(\tilde{\mathbf{r}})=\varepsilon_{o}\left[\langle\mathbf{E}(\tilde{\mathbf{r}}, t) \mathbf{E}(\tilde{\mathbf{r}}, t)\rangle-\frac{1}{2} \overline{\bar{I}}\left\langle\mathbf{E}^{2}(\tilde{\mathbf{r}}, t)\right\rangle\right], \\
& \overline{\bar{\sigma}}^{m}(\tilde{\mathbf{r}})=\mu_{o}\left[\langle\mathbf{H}(\tilde{\mathbf{r}}, t) \mathbf{H}(\tilde{\mathbf{r}}, t)\rangle-\frac{1}{2} \overline{\bar{I}}\left\langle\mathbf{H}^{2}(\tilde{\mathbf{r}}, t)\right\rangle\right] .
\end{aligned}
$$

The traction at a location $\tilde{\mathbf{r}}$ on the surface of object 2 can be obtained by evaluating $\mathbf{n}(\tilde{\mathbf{r}}) \cdot\left[\overline{\bar{\sigma}}^{e}(\tilde{\mathbf{r}})+\overline{\bar{\sigma}}^{m}(\tilde{\mathbf{r}})\right]$. The normal component of the traction at $\tilde{\mathbf{r}}$ is given by

$$
\begin{aligned}
F_{1 \rightarrow 2}(\tilde{\mathbf{r}}) & =\mathbf{n}_{2}(\tilde{\mathbf{r}}) \cdot\left[\overline{\bar{\sigma}}^{e}(\tilde{\mathbf{r}})+\overline{\bar{\sigma}}^{m}(\tilde{\mathbf{r}})\right] \cdot \mathbf{n}_{2}(\tilde{\mathbf{r}}) \\
& =\int_{0}^{\infty} \frac{d \omega}{2 \pi} \frac{2 \omega}{c^{2}} \Theta\left(\omega, T_{1}\right) \oint_{S_{1}} d s(\mathbf{r}) S_{1 \rightarrow 2}^{(m)}(\tilde{\mathbf{r}}, \mathbf{r}, \omega),
\end{aligned}
$$

where $\oint_{S_{1}} d s(\mathbf{r}) S_{1 \rightarrow 2}^{(m)}(\tilde{\mathbf{r}}, \mathbf{r}, \omega)$ is given by

$$
\begin{aligned}
\oint_{S_{1}} d s(\mathbf{r}) S_{1 \rightarrow 2}^{(m)}(\tilde{\mathbf{r}}, \mathbf{r}, \omega)= & \mathbf{n}_{2}(\tilde{\mathbf{r}}) \cdot \overline{\overline{\mathcal{E}}}^{(1)}(\tilde{\mathbf{r}}) \cdot \mathbf{n}_{2}(\tilde{\mathbf{r}})-\frac{\mathcal{E}_{p p}^{(1)}(\tilde{\mathbf{r}})}{2} \\
& +\mathbf{n}_{2}(\tilde{\mathbf{r}}) \cdot \overline{\overline{\mathcal{H}}}^{(1)}(\tilde{\mathbf{r}}) \cdot \mathbf{n}_{2}(\tilde{\mathbf{r}})-\frac{\mathcal{H}_{p p}^{(1)}(\tilde{\mathbf{r}})}{2}
\end{aligned}
$$

and $\mathcal{E}_{p p}^{(1)}(\tilde{\mathbf{r}})=\operatorname{Tr} \overline{\overline{\mathcal{E}}}^{(1)}(\tilde{\mathbf{r}})$. For the case of two half-spaces, the contribution to van der Waals pressure at any location on the surface of half-space 2 due to half-space 1 can be calculated by putting $\mathbf{n}_{2}(\tilde{\mathbf{r}})=-\hat{z}$. The term $\mathbf{n}_{2}(\tilde{\mathbf{r}}) \cdot \overline{\overline{\mathcal{E}}}^{(1)}(\tilde{\mathbf{r}}) \cdot \mathbf{n}_{2}(\tilde{\mathbf{r}})-\frac{1}{2} \mathcal{E}_{p p}^{(1)}(\tilde{\mathbf{r}})$ is then $-\frac{1}{2}\left(\mathcal{E}_{x x}^{(1)}+\mathcal{E}_{y y}^{(1)}-\mathcal{E}_{z z}^{(1)}\right)$, where the $x$ and $y$ axes are in the plane of the interface and the $z$ axis is out of plane (see Fig. 1). Similarly, the other term $\mathbf{n}_{2}(\tilde{\mathbf{r}}) \cdot \overline{\overline{\mathcal{H}}}^{(1)}(\tilde{\mathbf{r}}) \cdot \mathbf{n}_{2}(\tilde{\mathbf{r}})-\frac{1}{2} \mathcal{H}_{p p}^{(1)}(\tilde{\mathbf{r}})=$ $-\frac{1}{2}\left(\mathcal{H}_{x x}^{(1)}+\mathcal{H}_{y y}^{(1)}-\mathcal{H}_{z z}^{(1)}\right)$.

The dyadic Green's functions in Eq. (8) and those required to evaluate Eq. (10) for a planar multilayered configuration are given by $\overline{\bar{G}}_{e}(\mathbf{r}, \tilde{\mathbf{r}})=\overline{\bar{G}}^{(o)}(\mathbf{r}, \tilde{\mathbf{r}})+\overline{\bar{G}}_{e}^{(s c)}(\mathbf{r}, \tilde{\mathbf{r}})$ and $\overline{\bar{G}}_{m}(\mathbf{r}, \tilde{\mathbf{r}})=$ $\overline{\bar{G}}^{(o)}(\mathbf{r}, \tilde{\mathbf{r}})+\overline{\bar{G}}_{m}^{(s c)}(\mathbf{r}, \tilde{\mathbf{r}})$, where $\overline{\bar{G}}^{(o)}(\mathbf{r}, \tilde{\mathbf{r}})$, the DGF in a homogeneous medium, is given by $[11,24]$

$$
\begin{aligned}
& \overline{\bar{G}}^{(o)}(\mathbf{r}, \tilde{\mathbf{r}}) \\
& =\frac{i}{8 \pi^{2}} \int_{0}^{\infty} \int_{0}^{2 \pi} \frac{d k_{\rho} k_{\rho} d \phi}{k_{z v}} e^{i \boldsymbol{k}_{\rho} \cdot(\boldsymbol{\rho}-\tilde{\boldsymbol{\rho}})} \\
& \times \sum_{\mu=s, p}\left\{\begin{array}{lrr}
\hat{x}^{(\mu)}\left(+k_{z v}\right) \hat{x}^{(\mu)}\left(+k_{z v}\right) e^{i(z-\tilde{z}) k_{z v}} & \text { if } \quad z>\tilde{z} \\
\hat{x}^{(\mu)}\left(-k_{z v}\right) \hat{x}^{(\mu)}\left(-k_{z v}\right) e^{i(\tilde{z}-z) k_{z v}} & \text { if } \quad z<\tilde{z}
\end{array}\right.
\end{aligned}
$$

and the scattered DGFs $\overline{\bar{G}}_{e}^{(s c)}(\mathbf{r}, \tilde{\mathbf{r}})$ and $\overline{\bar{G}}_{m}^{(s c)}(\mathbf{r}, \tilde{\mathbf{r}})$ are given by

$$
\begin{aligned}
\overline{\bar{G}}_{e}^{(s c)}(\mathbf{r}, \tilde{\mathbf{r}})= & \frac{i}{8 \pi^{2}} \int_{0}^{\infty} \int_{0}^{2 \pi} \frac{d k_{\rho} k_{\rho} d \phi}{k_{z v}} \sum_{\mu=s, p} \frac{e^{i \boldsymbol{k}_{\rho} \cdot(\boldsymbol{\rho}-\tilde{\boldsymbol{\rho}})}}{\widetilde{D}^{(\mu)}} \\
& \times \sum_{\nu= \pm 1} \sum_{\xi= \pm 1} C_{\nu, \xi}^{(\mu)} \hat{x}^{(\mu)}\left(\nu k_{z v}\right) \hat{x}^{(\mu)}\left(\xi k_{z v}\right) e^{i(\nu z-\xi \tilde{z}) k_{z v}} \\
\overline{\bar{G}}_{m}^{(s c)}(\mathbf{r}, \tilde{\mathbf{r}})= & \frac{i}{8 \pi^{2}} \int_{0}^{\infty} \int_{0}^{2 \pi} \frac{d k_{\rho} k_{\rho} d \phi}{k_{z v}} \sum_{\mu=s, p} \frac{e^{i \boldsymbol{k}_{\rho} \cdot(\boldsymbol{\rho}-\tilde{\boldsymbol{\rho}})}}{\widetilde{D}^{\left(\mu^{\prime}\right)}} \\
& \times \sum_{\nu= \pm 1} \sum_{\xi= \pm 1} C_{\nu, \xi}^{\left(\mu^{\prime}\right)} \hat{x}^{(\mu)}\left(\nu k_{z v}\right) \hat{x}^{(\mu)}\left(\xi k_{z v}\right) e^{i(\nu z-\xi \tilde{z}) k_{z v}},
\end{aligned}
$$

where $\boldsymbol{\rho}=x \hat{x}+y \hat{y}, \quad \tilde{\boldsymbol{\rho}}=\tilde{x} \hat{x}+\tilde{y} \hat{y}, \quad \boldsymbol{k}_{\rho}=k_{\rho} \hat{k}_{\rho}=k_{x} \hat{x}+$ $k_{y} \hat{y}, \quad \hat{x}^{(s)}\left( \pm k_{z v}\right)=\hat{k}_{\rho} \times \hat{z}=\left(k_{y} \hat{x}-k_{x} \hat{y}\right) / k_{\rho}, \quad \hat{x}^{(p)}\left( \pm k_{z v}\right)=$ $\left(\mp k_{z v} \hat{k}_{\rho}+k_{\rho} \hat{z}\right) / k_{0}, \mu^{\prime}=p$ if $\mu=s$ and $\mu^{\prime}=s$ if $\mu=p$, and $\widetilde{D}^{(\mu)}=1-\widetilde{R}_{v 1}^{(\mu)} \widetilde{R}_{v 2}^{(\mu)} e^{i 2 k_{z v} d}$ and

$$
C_{\nu, \xi}^{(\mu)}= \begin{cases}\widetilde{R}_{v 1}^{(\mu)} & \text { if } \quad v=1, \xi=-1 \\ \widetilde{R}_{v 2}^{(\mu)} e^{i 2 k_{z v} d} & \text { if } \quad v=-1, \xi=1 \\ \widetilde{R}_{v 1}^{(\mu)} \widetilde{R}_{v 2}^{(\mu)} e^{i 2 k_{z v} d} & \text { if } \quad v=\xi .\end{cases}
$$

The position vectors $\mathbf{r}=\boldsymbol{\rho}$ and $\tilde{\mathbf{r}}=\tilde{\boldsymbol{\rho}}+d \hat{z}$ lie on the interfaces of half-spaces 1 and 2 with vacuum, respectively.

\section{DECOMPOSITION INTO SURFACE CONTRIBUTIONS}

We can give the following physical interpretations to $S_{1 \rightarrow 2}^{(e)}(\tilde{\mathbf{r}}, \mathbf{r}, \omega)$ and $S_{1 \rightarrow 2}^{(m)}(\tilde{\mathbf{r}}, \mathbf{r}, \omega): S_{1 \rightarrow 2}^{(e)}(\tilde{\mathbf{r}}, \mathbf{r}, \omega) d s(\mathbf{r}) d \omega$ and $S_{1 \rightarrow 2}^{(m)}(\tilde{\mathbf{r}}, \mathbf{r}, \omega) d s(\mathbf{r}) d \omega$ are the contributions to energy flow rate per unit surface area and pressure, respectively, at $\tilde{\mathbf{r}}$ from a patch of area $d s(\mathbf{r})$ at the position vector $\mathbf{r} \in S_{1}$ from the frequency interval $(\omega, \omega+d \omega)$. In reality, the sources for these interactions are spread throughout half-space 1 but the effect of these sources at $\tilde{\mathbf{r}}$ can be decomposed into contributions from the surface of half-space 1. In Ref. [11], the authors have derived expressions for radiative energy and momentum transfer between two objects in terms of volume integrals and corresponding surface integrals of dyadic Green's function of the vector Helmholtz equation (satisfying appropriate boundary conditions). One of the advantages of using the surface integral formalism is the potential reduction in computational cost from a three-dimensional domain to two dimensions.

Without loss of generality, $\tilde{\mathbf{r}}$ will be chosen such that it lies on the $z$ axis. The origin of the coordinate system is chosen to lie on the surface of half-plane 1 . Hence the coordinate of $\tilde{\mathbf{r}}$ is $(0,0, d)$. Since the configuration has translational symmetry in the $x-y$ plane, the contributions to the energy transfer rate and pressure at $\tilde{\mathbf{r}}$ are axisymmetric. Hence, we choose $\mathbf{r}=\rho \cos \theta \hat{x}+\rho \sin \theta \hat{y}$, as shown in Fig. 1, where $\rho$ and $\theta$ are coordinates of $\mathbf{r}$ in plane polar coordinates. $d s(\mathbf{r})$ has a magnitude $\rho d \rho d \theta$. The in-plane wave vector $\boldsymbol{k}_{\boldsymbol{\rho}}$ is defined as $\boldsymbol{k}_{\rho}=k_{\rho}(\hat{x} \cos \phi+\hat{y} \sin \phi)$. 
From the expressions for the DGFs in Eqs. (12)-(14), we can see that computing $S_{1 \rightarrow 2}^{(e)}(\tilde{\mathbf{r}}, \mathbf{r}, \omega)$ and $S_{1 \rightarrow 2}^{(m)}(\tilde{\mathbf{r}}, \mathbf{r}, \omega)$ will involve integrals of the following form:

$$
\begin{aligned}
& {\left[\int_{0}^{\infty} \int_{0}^{2 \pi} \frac{d k_{\rho} k_{\rho} d \phi}{k_{z v}} e^{i k_{\rho} \cdot \rho} f\left(k_{z v}\right) \hat{x}^{(\mu)}\left(v k_{z v}\right) \hat{x}^{(\mu)}\left(\xi k_{z v}\right)\right]^{*}} \\
& \quad \cdot\left[\int_{0}^{\infty} \int_{0}^{2 \pi} \frac{d \bar{k}_{\rho} \bar{k}_{\rho} d \bar{\phi}}{\bar{k}_{z v}} e^{i \bar{k}_{\rho} \cdot \rho} g\left(\bar{k}_{z v}\right) \hat{x}^{(\bar{\mu})}\left(\bar{v} \bar{k}_{z v}\right) \hat{x}^{(\bar{\mu})}\left(\bar{\xi} \bar{k}_{z v}\right)\right] .
\end{aligned}
$$

In Eq. (16), the functions $f\left(k_{z v}\right)$ and $g\left(\bar{k}_{z v}\right)$ are functions of the reflection coefficients as well as the gap between the two half-spaces. The vectors $\hat{x}^{(\mu)}\left(v k_{z v}\right), \hat{x}^{(\mu)}\left(\xi k_{z v}\right), \hat{x}^{(\bar{\mu})}\left(\bar{v}_{z v}\right)$, and $\hat{x}^{(\bar{\mu})}\left(\bar{\xi} \bar{k}_{z v}\right), \quad$ defined after Eq. (14), could be functions of $\phi$ or $\bar{\phi}$ too. It should also be kept in mind that dot products of vectors associated with different polarizations could arise (when $\mu \neq \bar{\mu}$ ). This is not surprising since we are, at this stage, considering the interactions between parts of the two half-spaces. When this result is integrated appropriately from $\rho=0$ to $\rho=\infty$, the dot products between cross polarizations drop out to give results similar to Eq. (2) or Eq. (3). The calculations of $S_{1 \rightarrow 2}^{(e)}(\tilde{\mathbf{r}}, \mathbf{r}, \omega)$ and $S_{1 \rightarrow 2}^{(m)}(\tilde{\mathbf{r}}, \mathbf{r}, \omega)$ involve computing the trace of a dyad [Eq. (8)] or finding $-\frac{1}{2}\left(\mathcal{E}_{x x}^{(1)}+\mathcal{E}_{y y}^{(1)}-\mathcal{E}_{z z}^{(1)}\right.$ and $\left.-\frac{1}{2} \mathcal{H}_{x x}^{(1)}+\mathcal{H}_{y y}^{(1)}-\mathcal{H}_{z z}^{(1)}\right)$. Because of these operations, $S_{1 \rightarrow 2}^{(e)}(\tilde{\mathbf{r}}, \mathbf{r}, \omega)$ and $S_{1 \rightarrow 2}^{(m)}(\tilde{\mathbf{r}}, \mathbf{r}, \omega)$ can be written in the form

$$
\begin{aligned}
& S_{1 \rightarrow 2}^{(\alpha)}(\tilde{\mathbf{r}}, \mathbf{r}, \omega) \\
& =\int_{0}^{\infty} \int_{0}^{2 \pi} \frac{d k_{\rho} k_{\rho} d \phi}{k_{z v}^{*}} \int_{0}^{\infty} \int_{0}^{2 \pi} \frac{d \bar{k}_{\rho} \bar{k}_{\rho} d \bar{\phi}}{\bar{k}_{z v}} e^{i\left(\boldsymbol{k}_{\rho}-\bar{k}_{\rho}\right) \cdot \rho} \\
& \quad \times\left[\left(\frac{k_{x} \bar{k}_{x} \pm k_{y} \bar{k}_{y}}{k_{\rho} \bar{k}_{\rho}}\right)^{2}[\cdots]+\left(\frac{k_{x} \bar{k}_{x}+k_{y} \bar{k}_{y}}{k_{\rho} \bar{k}_{\rho}}\right)[\cdots]\right],
\end{aligned}
$$

where $\alpha=e, m$, and the coordinates of $\tilde{\mathbf{r}}$ and $\mathbf{r}$ are $(0,0, d)$ and $(\rho \cos \theta, \rho \sin \theta, 0)$, respectively. The terms within [ $\cdots]$ denote products of different $f\left(k_{z v}\right)$ and $g\left(\bar{k}_{z v}\right)$ functions. It is important to keep in mind that the terms represented as $[\cdots]$ are functions of only $k_{\rho}, \bar{k}_{\rho}$ and not of $\phi$ or $\bar{\phi}$.

Since $\boldsymbol{k}_{\rho}=k_{\rho}(\hat{x} \cos \phi+\hat{y} \sin \phi)$ and $\overline{\boldsymbol{k}}_{\rho}=\bar{k}_{\rho}(\hat{x} \cos \bar{\phi}+$ $\hat{y} \sin \bar{\phi}$ ), we can write

$$
\begin{aligned}
& \frac{k_{x} \bar{k}_{x}+k_{y} \bar{k}_{y}}{k_{\rho} \bar{k}_{\rho}}=\cos (\phi-\bar{\phi}), \\
& \frac{k_{x} \bar{k}_{x}-k_{y} \bar{k}_{y}}{k_{\rho} \bar{k}_{\rho}}=\sin (\phi-\bar{\phi}),
\end{aligned}
$$

$$
\left(\frac{k_{x} \bar{k}_{x} \pm k_{y} \bar{k}_{y}}{k_{\rho} \bar{k}_{\rho}}\right)^{2}=\frac{1}{2}[1 \pm \cos 2(\phi-\bar{\phi})] .
$$

Using the relation $J_{n}(x)=\frac{i^{-n}}{2 \pi} \int_{0}^{2 \pi} d \phi e^{i(x \cos \phi+n \phi)}$, the integration over $\phi$ or $\bar{\phi}$ in Eq. (17) yields the following relations:

$$
\begin{aligned}
& \int_{0}^{2 \pi} \int_{0}^{2 \pi} d \phi d \bar{\phi} e^{i\left(k_{\rho}-\bar{k}_{\rho}\right) \cdot \rho}[1 \pm \cos 2(\phi-\bar{\phi})] \\
& =4 \pi^{2}\left[J_{0}\left(k_{\rho} \rho\right) J_{0}\left(\bar{k}_{\rho} \rho\right) \pm J_{2}\left(k_{\rho} \rho\right) J_{2}\left(\bar{k}_{\rho} \rho\right)\right], \\
& \int_{0}^{2 \pi} \int_{0}^{2 \pi} d \phi d \bar{\phi} e^{i\left(k_{\rho}-\bar{k}_{\rho}\right) \cdot \rho} \cos (\phi-\bar{\phi})=4 \pi^{2} J_{1}\left(k_{\rho} \rho\right) J_{1}\left(\bar{k}_{\rho} \rho\right) .
\end{aligned}
$$

The position vector $\mathbf{r}$ now appears only in the argument of the Bessel functions as $\rho$ in Eqs. (19a) and (19b). By using the identity

$$
\int_{0}^{\infty} d \rho \rho J_{n}\left(k_{\rho} \rho\right) J_{n}\left(\bar{k}_{\rho} \rho\right)=\frac{1}{k_{\rho} \bar{k}_{\rho}} \delta\left(k_{\rho}-\bar{k}_{\rho}\right),
$$

we have shown that integration of $S_{1 \rightarrow 2}^{(e)}(\tilde{\mathbf{r}}, \mathbf{r}, \omega)$ and $\left(2 \omega / c^{2}\right) S_{1 \rightarrow 2}^{(m)}(\tilde{\mathbf{r}}, \mathbf{r}, \omega)$ over $S_{1}$ yield exactly the same results as Eqs. (2) and (3), respectively.

In order to simplify the terms within $[\cdots]$ in Eq. (17), we define a general form of the integral over $q_{\rho}$ as

$$
\begin{aligned}
& I_{n, \pm \pm}^{a, b,(\mu)}=\int_{0}^{\infty} d q_{\rho} q_{\rho}^{a} q_{z v}^{b} J_{n}\left(q_{\rho} k_{0} \rho\right) e^{i q_{z v} k_{0} d}\left(1+f_{ \pm \pm}^{(\mu)}\right), \\
& I_{n, \pm \mp}^{a, b,(\mu)}=\int_{0}^{\infty} d q_{\rho} q_{\rho}^{a} q_{z v}^{b} J_{n}\left(q_{\rho} k_{0} \rho\right) e^{i q_{z v} k_{0} d}\left(1+f_{ \pm \mp}^{(\mu)}\right),
\end{aligned}
$$

where $q_{\rho}=k_{\rho} / k_{0}$ is a nondimensionalized in-plane wave vector, $q_{z v}=k_{z v} / k_{0}$ is the nondimensionalized $z$-component wave vector, $1+f_{ \pm \pm}^{(\mu)}=\left(1 \pm \widetilde{R}_{v 1}^{(\mu)}\right)\left(1 \pm \widetilde{R}_{v 2}^{(\mu)}\right) / \widetilde{D}^{(\mu)}$, $1+f_{ \pm \mp}^{(\mu)}=\left(1 \pm \widetilde{R}_{v 1}^{(\mu)}\right)\left(1 \mp \widetilde{R}_{v 2}^{(\mu)}\right) / \widetilde{D}^{(\mu)}, \quad$ and $\quad \widetilde{D}^{(\mu)}=$ $1-\widetilde{R}_{v 1}^{(\mu)} \widetilde{R}_{v 2}^{(\mu)} e^{i 2 q_{z v} k_{0} d}$. The functions $f_{ \pm \pm}^{(\mu)}$ and $f_{ \pm \mp}^{(\mu)}$ are given by

$$
\begin{aligned}
f_{ \pm \pm}^{(\mu)} & =\frac{ \pm \widetilde{R}_{v 1}^{(\mu)} \pm \widetilde{R}_{v 2}^{(\mu)}+\widetilde{R}_{v 1}^{(\mu)} \widetilde{R}_{v 2}^{(\mu)}\left(1+e^{i 2 q_{z v} k_{0} d}\right)}{\widetilde{D}^{(\mu)}} \\
f_{ \pm \mp}^{(\mu)} & =\frac{ \pm \widetilde{R}_{v 1}^{(\mu)} \mp \widetilde{R}_{v 2}^{(\mu)}-\widetilde{R}_{v 1}^{(\mu)} \widetilde{R}_{v 2}^{(\mu)}\left(1-e^{i 2 q_{z v} k_{0} d}\right)}{\widetilde{D}^{(\mu)}}
\end{aligned}
$$

By substituting the definitions from Eqs. (21) into Eq. (8), we obtain the following equation for $S_{1 \rightarrow 2}^{(e)}(\tilde{\mathbf{r}}, \mathbf{r}, \omega)$ :

$$
\begin{aligned}
S_{1 \rightarrow 2}^{(e)}(\tilde{\mathbf{r}}, \mathbf{r}, \omega)= & k_{0}^{4} \operatorname{Re} \sum_{\mu=s, p}\left[I_{0,++}^{1,-1,(\mu) *}\left[I_{0,--}^{1,1,(\mu)}+I_{0,++}^{1,1,\left(\mu^{\prime}\right)}\right]+I_{0,+-}^{1,0,(\mu) *}\left[I_{0,-+}^{1,0,(\mu)}+I_{0,+-}^{1,0,\left(\mu^{\prime}\right)}\right]\right. \\
& \left.+I_{2,++}^{1,-1,(\mu) *}\left[I_{2,--}^{1,1,(\mu)}-I_{2,++}^{1,1,\left(\mu^{\prime}\right)}\right]+I_{2,+-}^{1,0,(\mu) *}\left[I_{2,-+}^{1,0,(\mu)}-I_{2,+-}^{1,0,\left(\mu^{\prime}\right)}\right]\right]
\end{aligned}
$$

where $\mu^{\prime}=p$ if $\mu=s$ and $\mu^{\prime}=s$ if $\mu=p$. 
Similarly, by substituting the definitions from Eqs. (21) into Eq. (10), we obtain the following equation for $S_{1 \rightarrow 2}^{(m)}(\tilde{\mathbf{r}}, \mathbf{r}, \omega)$ :

$$
\begin{aligned}
S_{1 \rightarrow 2}^{(m)}(\tilde{\mathbf{r}}, \mathbf{r}, \omega)= & k_{0}^{3} \operatorname{Re} \sum_{\mu=s, p}\left[I_{0,++}^{1,-1,(\mu) *}\left[I_{0,+-}^{1,0,(\mu)}+I_{0,-+}^{1,0,\left(\mu^{\prime}\right)}\right]+I_{0,--}^{1,1,(\mu) *}\left[I_{0,-+}^{1,0,(\mu)}+I_{0,+-}^{1,0,\left(\mu^{\prime}\right)}\right]\right. \\
& \left.+I_{2,++}^{1,-1,(\mu) *}\left[I_{2,+-}^{1,0,(\mu)}-I_{2,-+}^{1,0,\left(\mu^{\prime}\right)}\right]+I_{2,--}^{1,1,(\mu) *}\left[I_{2,-+}^{1,0,(\mu)}-I_{2,+-}^{1,0,\left(\mu^{\prime}\right)}\right]-2 I_{1,++}^{2,-1,(\mu) *} I_{1,+-}^{2,0,(\mu)}\right],
\end{aligned}
$$

where $\mu^{\prime}=p$ if $\mu=s$ and $\mu^{\prime}=s$ if $\mu=p$.

\section{A. Evaluating $S_{1 \rightarrow 2}^{(e)}(\tilde{\mathbf{r}}, \mathbf{r}, \omega)$ and $S_{1 \rightarrow 2}^{(m)}(\tilde{\mathbf{r}}, \mathrm{r}, \omega)$}

To calculate $S_{1 \rightarrow 2}^{(e)}(\tilde{\mathbf{r}}, \mathbf{r}, \omega)$ and $S_{1 \rightarrow 2}^{(m)}(\tilde{\mathbf{r}}, \mathbf{r}, \omega)$, we take the two half-spaces to be silica. The real and imaginary parts of the dielectric function of silica [17] are shown in Fig. 2(a). We plot the spectral contributions of radiative energy transfer and van der Waals pressure in Fig. 2(b), $\oint_{S_{1}} d s(\mathbf{r}) S_{1 \rightarrow 2}^{(e)}(\tilde{\mathbf{r}}, \mathbf{r}, \omega)=T_{1 \rightarrow 2}^{(e)}(\omega)$ and $\frac{2 \omega}{c^{2}} \oint_{S_{1}} d s(\mathbf{r}) S_{1 \rightarrow 2}^{(m)}(\tilde{\mathbf{r}}, \mathbf{r}, \omega)=T_{1 \rightarrow 2}^{(m)}(\omega)$, using Eqs. (2) and (3), respectively, in order to identify frequency intervals of interest in evaluation of $S_{1 \rightarrow 2}^{(e)}(\tilde{\mathbf{r}}, \mathbf{r}, \omega)$ and $S_{1 \rightarrow 2}^{(m)}(\tilde{\mathbf{r}}, \mathbf{r}, \omega)$. The calculations are done for $d=10 \mathrm{~nm}, T_{1}=300 \mathrm{~K}$, and $T_{2}=0 \mathrm{~K}$.

We can see from Fig. 2(b) that $T_{1 \rightarrow 2}^{(e)}(\omega)$ has contributions from lower frequencies compared to $T_{1 \rightarrow 2}^{(m)}(\omega)$. This is because only temperature dependent fluctuations give rise to net radiative energy transfer, whereas van der Waals pressure at any location has contributions from zero-point fluctuations as well [25]. For far-field thermal radiation, approximately $97 \%$ of the total energy transfer lies in $\frac{1}{5} \frac{2 \pi c}{\lambda_{T}} \leqslant \omega \leqslant 5 \frac{2 \pi c}{\lambda_{T}}$, where $\lambda_{T}=1.27 \frac{\hbar c}{k_{B} T}$ is wavelength corresponding to the peak of the blackbody spectrum [26]. The contribution to van der Waals pressure is skewed towards the ultraviolet portion of the electromagnetic spectrum because of the zero-point fluctations. Using $\omega_{c}=2 \times 10^{16} \mathrm{rad} / \mathrm{s}$ and $\omega_{c}=2 \pi c / \lambda_{T}$ as the characteristic frequencies for van der Waals pressure and energy transfer, respectively, we see that a nondimensional size

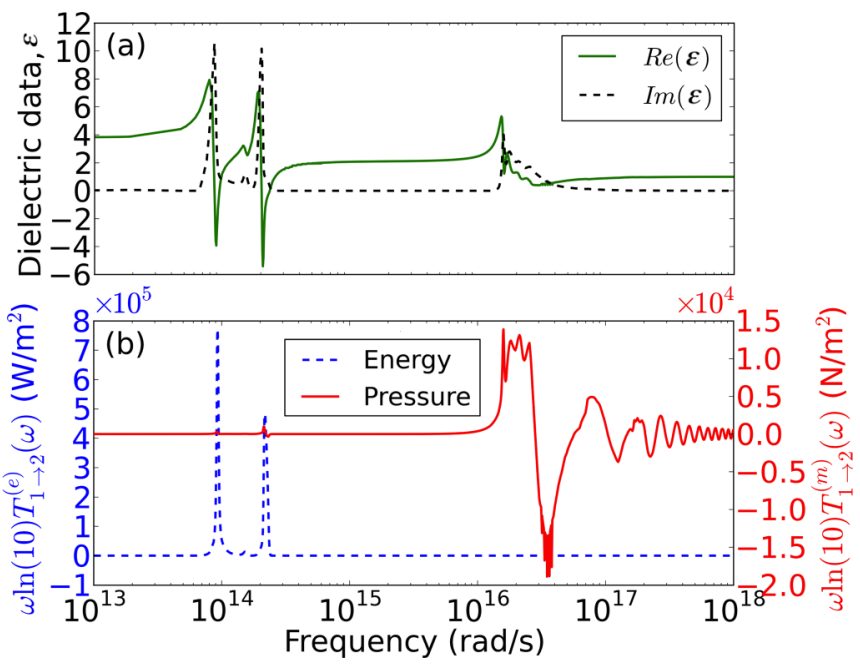

FIG. 2. (Color online) (a) Real and imaginary parts of dielectric function of silica. (b) Spectral contributions of radiative energy transfer (dashed line) and van der Waals pressure (solid line). Because of the logarithmic $x$ axis, we have plotted $\omega \ln (10) T_{1 \rightarrow 2}^{(\alpha)}(\alpha=e, m)$ so that the area under the plotted graph is proportional to the integral of $T_{1 \rightarrow 2}^{(\alpha)}$. parameter, defined as $\omega_{c} d / c$ is $\gtrsim 0.6$ for van der Waals pressure and $\approx 0.006$ for energy transfer. Hence, the computation for van der Waals pressure is expected to be much tougher than for energy transfer.

$S_{1 \rightarrow 2}^{(e)}(\tilde{\mathbf{r}}, \mathbf{r}, \omega)$ is evaluated directly by computing Eq. (23). To compute $S_{1 \rightarrow 2}^{(m)}(\tilde{\mathbf{r}}, \mathbf{r}, \omega)$, we split it into two parts. We write $S_{1 \rightarrow 2}^{(m)}(\tilde{\mathbf{r}}, \mathbf{r}, \omega)=S_{1 \rightarrow 2}^{(m) h}(\tilde{\mathbf{r}}, \mathbf{r}, \omega)+S_{1 \rightarrow 2}^{(m) s c}(\tilde{\mathbf{r}}, \mathbf{r}, \omega) . \quad S_{1 \rightarrow 2}^{(m) h}(\tilde{\mathbf{r}}, \mathbf{r}, \omega)$ is the contribution to $S_{1 \rightarrow 2}^{(m)}(\tilde{\mathbf{r}}, \mathbf{r}, \omega)$ when the half-spaces are replaced by vacuum. $S_{1 \rightarrow 2}^{(m) s c}(\tilde{\mathbf{r}}, \mathbf{r}, \omega)$ contains the effect of reflections at the interfaces between vacuum and half-spaces as well as the $d$-dependent part of $S_{1 \rightarrow 2}^{(m)}(\tilde{\mathbf{r}}, \mathbf{r}, \omega) . S_{1 \rightarrow 2}^{(m) s c}(\tilde{\mathbf{r}}, \mathbf{r}, \omega)$ can be written as

$$
\begin{aligned}
& S_{1 \rightarrow 2}^{(m) s c}(\tilde{\mathbf{r}}, \mathbf{r}, \omega) \\
& =k_{0}^{3} \operatorname{Re}\left[2 \sum _ { \mu = s , p } \left[\left(I_{0}^{1,-1}+I_{0}^{1,1}\right)^{*} X_{0}^{(\mu)}+\left(I_{2}^{1,-1}-I_{2}^{1,1}\right)^{*} Z_{2}^{(\mu)}\right.\right. \\
& \left.\quad+I_{0}^{1,0 *} Y_{0}^{(\mu)}-I_{1}^{2,0 *} V_{1}^{(\mu)}-I_{1}^{2,-1 *} U_{1}^{(\mu)}-U_{1}^{(\mu) *} V_{1}^{(\mu)}\right] \\
& \quad+\left(X_{0}^{(s)}+X_{0}^{(p)}\right)^{*}\left(Y_{0}^{(s)}+Y_{0}^{(p)}\right) \\
& \left.\quad+\left(X_{2}^{(s)}-X_{2}^{(p)}\right)^{*}\left(Y_{2}^{(s)}-Y_{2}^{(p)}\right)\right]
\end{aligned}
$$

where $I_{n}^{a, b}$ are the functions with homogeneous contributions, given by [27]

$$
\begin{aligned}
I_{0}^{1,-1}= & -i \frac{e^{i k_{0} R}}{k_{0} R}, \\
I_{0}^{1,0}= & -i \frac{e^{i k_{0} R}}{k_{0} R}\left(1+\frac{i}{k_{0} R}\right) \frac{d}{R}, \\
I_{0}^{1,1}= & -i \frac{e^{i k_{0} R}}{k_{0} R}\left[\frac{d^{2}}{R^{2}}\left(1+\frac{i 3}{k_{0} R}-\frac{3}{k_{0}^{2} R^{2}}\right)\right. \\
& \left.-\frac{i}{k_{0} R}\left(1+\frac{i}{k_{0} R}\right)\right], \\
I_{2}^{1,-1}= & \frac{2}{k_{0}^{2} \rho^{2}}\left(e^{i k_{0} d}-e^{i k_{0} R}\right)+i \frac{e^{i k_{0} R}}{k_{0} R}, \\
I_{2}^{1,0}= & \frac{2}{k_{0}^{2} \rho^{2}}\left(e^{i k_{0} d}-\frac{d}{R} e^{i k_{0} R}\right)+i \frac{e^{i k_{0} R}}{k_{0} R}\left(1+\frac{i}{k_{0} R}\right) \frac{d}{R}, \\
I_{2}^{1,1}= & \frac{2}{k_{0}^{2} \rho^{2}}\left(e^{i k_{0} d}-e^{i k_{0} R}\right)+i \frac{e^{i k_{0} R}}{k_{0} R} \\
& \times\left[-\frac{i^{2}}{k_{0} R}+\frac{3}{k_{0}^{2} R^{2}}+\frac{d^{2}}{R^{2}}\left(1+\frac{i 3}{k_{0} R}-\frac{3}{k_{0}^{2} R^{2}}\right)\right],
\end{aligned}
$$




$$
\begin{aligned}
I_{1}^{2,0} & =-\frac{e^{i k_{0} R}}{k_{0} R}\left(1+\frac{i 3}{k_{0} R}-\frac{3}{k_{0}^{2} R^{2}}\right) \frac{\rho}{R} \frac{d}{R}, \\
I_{1}^{2,-1} & =-\frac{e^{i k_{0} R}}{k_{0} R}\left(1+\frac{i}{k_{0} R}\right) \frac{\rho}{R}
\end{aligned}
$$

and the functions with scattering parts are given by

$$
\begin{aligned}
X_{n}^{(\mu)} & =\int_{0}^{\infty} d q_{\rho} q_{\rho} J_{n}\left(q_{\rho} k_{0} \rho\right) e^{i q_{z v} k_{0} d}\left(\frac{f_{+-}^{(\mu)}+f_{-+}^{(\mu)}}{2}\right), \\
Y_{n}^{(\mu)} & =\int_{0}^{\infty} d q_{\rho} \frac{q_{\rho}}{q_{z v}} J_{n}\left(q_{\rho} k_{0} \rho\right) e^{i q_{z v} k_{0} d}\left(f_{++}^{(\mu)}+q_{z v}^{2} f_{--}^{(\mu)}\right) \\
Z_{n}^{(\mu)} & =\int_{0}^{\infty} d q_{\rho} q_{\rho} J_{n}\left(q_{\rho} k_{0} \rho\right) e^{i q_{z v} k_{0} d}\left(f_{+-}^{(\mu)}-f_{-+}^{(\mu)}\right), \\
U_{n}^{(\mu)} & =\int_{0}^{\infty} d q_{\rho} q_{\rho}^{2} J_{n}\left(q_{\rho} k_{0} \rho\right) e^{i q_{z v} k_{0} d} f_{+-}^{(\mu)} \\
V_{n}^{(\mu)} & =\int_{0}^{\infty} d q_{\rho} \frac{q_{\rho}^{2}}{q_{z v}} J_{n}\left(q_{\rho} k_{0} \rho\right) e^{i q_{z v} k_{0} d} f_{++}^{(\mu)}
\end{aligned}
$$

In Eq. (26), $R=|\mathbf{r}-\tilde{\mathbf{r}}|=\sqrt{\rho^{2}+d^{2}}$ is the distance between vectors $\mathbf{r}$ and $\tilde{\mathbf{r}}$.

To determine $S_{1 \rightarrow 2}^{(m) h}(\tilde{\mathbf{r}}, \mathbf{r}, \omega)$, we evaluate $S_{1 \rightarrow 2}^{(m)}(\tilde{\mathbf{r}}, \mathbf{r}, \omega)$ [Eq. (24)] when $\widetilde{R}_{v 1}^{(\mu)}=\widetilde{R}_{v 2}^{(\mu)}=0$. Substituting Eqs. (26a)(26h) into Eq. (24), we get $S_{1 \rightarrow 2}^{(m) h}(\tilde{\mathbf{r}}, \mathbf{r}, \omega)$ to be

$$
S_{1 \rightarrow 2}^{(m) h}(\tilde{\mathbf{r}}, \mathbf{r}, \omega)=\frac{8 k_{0}}{R^{2}} \frac{d^{3}}{R^{3}} .
$$

Though $S_{1 \rightarrow 2}^{(m) h}(\tilde{\mathbf{r}}, \mathbf{r}, \omega)$ is dependent on $d$ and $\rho$, we can evaluate the contribution from the whole of $S_{1}$ to give

$$
\oint_{S_{1}} d s(\mathbf{r}) S_{1 \rightarrow 2}^{(m) h}(\tilde{\mathbf{r}}, \mathbf{r}, \omega)=2 \pi \int_{0}^{\infty} d \rho \rho \frac{8 k_{0}}{R^{2}} \frac{d^{3}}{R^{3}}=\frac{16}{3} \pi k_{0} .
$$

As expected, integration of the homogeneous part $S_{1 \rightarrow 2}^{(m) h}(\tilde{\mathbf{r}}, \mathbf{r}, \omega)$ over the entire surface $S_{1}$ is independent of the spacing between the two half-spaces [28].

\section{B. Stationary phase method for computing high frequency integrals}

Equations (27a)-(27e) are one-dimensional integrals and are computed numerically using adaptive quadrature (we used the SciPy function quad for numerical integration). However, the oscillations at higher frequencies render this method unreliable. To overcome this problem, we use the stationary phase method for frequencies such that $k_{0} d \gg 1$ or $k_{0} \rho \gg 1$. The basic idea of the stationary phase method is that if the integrand is rapidly oscillating about a mean value of 0 , the contribution to the integration is small because of the cancellation of the positive and negative parts of the integrands. Therefore, most of the contribution to the integral is from the neighborhood of point $q_{\rho s}$ at which the oscillations are least. This point $q_{\rho s}$ is called the stationary phase point $[12,29]$ (the subscript $s$ indicates that at $q_{\rho s}$, the integrand attains a stationary phase). The integrals of the form $\int_{0}^{\infty} d q_{\rho} q_{\rho}^{a} q_{z v}^{b} J_{n}\left(q_{\rho} k_{0} \rho\right) e^{i q_{z v} k_{0} d} f\left(q_{\rho}\right)$ can be rewritten as

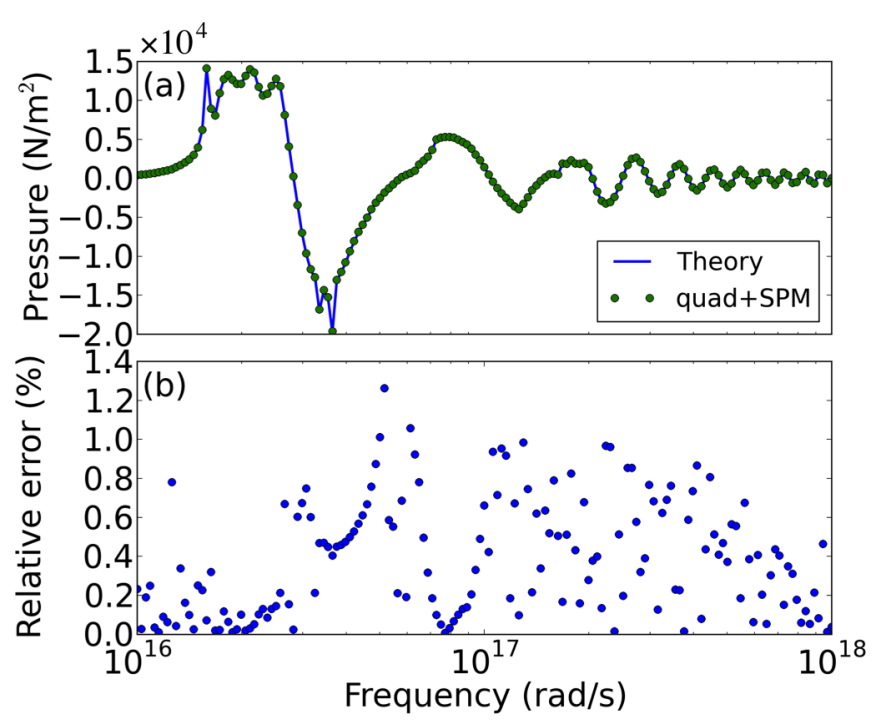

FIG. 3. (Color online) (a) Comparison of calculated van der Waals pressure contribution from one half-space to the other located $10 \mathrm{~nm}$ away by two methods: (1) direct integration over entire surface of half-space [using Eq. (3)] and (2) computing contribution from each $\rho$ and subsequent integration from $\rho=0$ to $\rho=\infty$. The legend "quad + SPM" stands for numerical integration using adaptive quadrature for $k_{0} R \leqslant 200$ and the stationary phase method for $k_{0} R \geqslant$ 200. As in Fig. 2, we are plotting $\omega \ln (10) T_{1 \rightarrow 2}^{(m)}$ (continuous line) and $\frac{2 \omega^{2}}{c^{2}} \ln (10) \oint_{S_{1}} d s(\mathbf{r}) S_{1 \rightarrow 2}^{(m)}(\tilde{\mathbf{r}}, \mathbf{r}, \omega)$ (circular markers), respectively. (b) Relative error between the two methods.

$\int_{-\infty}^{\infty} d q_{\rho} q_{\rho}^{a} q_{z v}^{b} \frac{1}{2} H_{n}^{(1)}\left(q_{\rho} k_{0} \rho\right) e^{i q_{z v} k_{0} d} f\left(q_{\rho}\right)$, where $f\left(q_{\rho}\right)$ can be any even function of $q_{\rho}$, as are any of the corresponding functions that appear in Eqs. (27a)-(27e). When $k_{0} \rho \gg n$, we can use the following limiting form for Hankel functions of the first kind (see Secs. 2.5 and 2.6 in Ref. [12]):

$$
H_{n}^{(1)}\left(q_{\rho} k_{0} \rho\right) \approx \sqrt{\frac{2}{\pi q_{\rho} k_{0} \rho}} \exp \left[i\left(q_{\rho} k_{0} \rho-\frac{n \pi}{2}-\frac{\pi}{4}\right)\right] .
$$

Using Eq. (30), we can see that the oscillations of the integrands in Eqs. (27a)-(27e) originate from the term $\exp \left[i\left(q_{\rho} k_{0} \rho+q_{z v} k_{0} d\right)\right]$. Here, the integration is in the form like Eq. (21). The stationary phase point $q_{\rho s}$ is calculated by setting $\frac{d}{d q_{\rho}}\left(q_{\rho} \rho+q_{z v} d\right)=0$. Hence $q_{\rho s}=\frac{\rho}{\sqrt{\rho^{2}+d^{2}}}=\frac{\rho}{R} \Rightarrow$ $q_{z v s}=\frac{d}{R}$. The stationary phase method approximation of an integral as $k_{0} R \gg 1$ can then be shown to be

$$
\int_{0}^{\infty} d q_{\rho} q_{\rho}^{a} q_{z v}^{b} J_{n}\left(q_{\rho} k_{0} \rho\right) e^{i q_{z v} k_{0} d} f\left(q_{\rho}\right) \approx I_{n}^{a, b} f\left(q_{\rho s}\right),
$$

where explicit forms for $I_{n}^{a, b}$ are given in Eqs. (26), and function $f\left(q_{\rho s}\right)$ is evaluated at stationary phase point.

To determine the accuracy of our computational method, we calculate $\frac{2 \omega}{c^{2}} \oint_{S_{1}} d s(\mathbf{r}) S_{1 \rightarrow 2}^{(m)}(\tilde{\mathbf{r}}, \mathbf{r}, \omega)$ for $d=10 \mathrm{~nm}$ and compare it with direct computation of $T_{1 \rightarrow 2}^{(m)}(\omega)$ through Eq. (3) [continuous line in Fig. 3(a) is from Eq. (3)]. As shown in Fig. 3(a), the spectral contributions to van der Waals pressure from surface patches are obtained by evaluating two integrations over $q_{\rho}$ at each $\rho$ and over $\rho$ in that order. Since direct numerical integration over $q_{\rho}$ is not reliable at either high frequencies or 
large values of $k_{0} R$, we choose a value $N$ such that we use the stationary phase method for $k_{0} R \geqslant N$ and use direct numercial (adaptive) integration only for smaller frequencies. We then use adaptive integration from $\rho_{\min }=0.001 d$ to $\rho_{\max }=100 d$ to retrieve $2 \pi \int_{\rho_{\min }}^{\rho_{\max }} d \rho \rho S_{1 \rightarrow 2}^{(\alpha)}(\tilde{\mathbf{r}}, \mathbf{r}, \omega) \approx \oint_{S_{1}} d s(\mathbf{r}) S_{1 \rightarrow 2}^{(\alpha)}(\tilde{\mathbf{r}}, \mathbf{r}, \omega)$ ( $\alpha=e, m)$ for each frequency. To generate the results in Fig. 3, $N$ is set to be 200. The relative error between the evaluation of $\frac{2 \omega}{c^{2}} \oint_{S_{1}} d s(\mathbf{r}) S_{1 \rightarrow 2}^{(m)}(\tilde{\mathbf{r}}, \mathbf{r}, \omega)$ and computation of $T_{1 \rightarrow 2}^{(m)}(\omega)$ through Eq. (3), as shown in Fig. 3(b), is $\lesssim 1.4 \%$ for $10^{16} \leqslant \omega \leqslant 10^{18}$ $\mathrm{rad} / \mathrm{s}$. We observed that a smaller value of $N$ reduces the computation time but also increases the error.

To further determine the accuracy of our computational procedure, we calculate the total energy flux and van der Waals pressure at $\tilde{\mathbf{r}}$ by (1) integrating contributions from different surface patches, and (2) direct integration of Eq. (2) or Eq. (3) along the real frequency axis [11,30]. Since we are evaluating the contribution from only one of the two (identical in properties) half-spaces, the van der Waals pressure at any location in the vacuum gap is half the value of equilibrium van der Waals pressure between the two half-spaces [30]. Hence, we also evaluate van der Waals pressure by Lifshitz theory, which involves summation of appropriate functions evaluated at Masubara frequencies on the imaginary frequency axis [15]. Each calculation of the total contributions from surface patches to energy flux and van der Waals pressure between two half-spaces requires one more integration over frequency $\omega$ besides the integrations over $q_{\rho}$ and $\rho$, which have been solved for generating Fig. 3. We finally evaluate $\int_{\omega_{\min }}^{\omega_{\max }} d \omega \oint_{S_{1}} d s(\mathbf{r}) S_{1 \rightarrow 2}^{(\alpha)}(\tilde{\mathbf{r}}, \mathbf{r}, \omega)$ at equally spaced frequencies with $\omega_{\min }=10^{13} \mathrm{rad} / \mathrm{s}$ and $\omega_{\max }=10^{15} \mathrm{rad} / \mathrm{s}$ for energy flux $(\alpha=e)$, and with $\omega_{\min }=10^{15} \mathrm{rad} / \mathrm{s}$ and $\omega_{\max }=10^{18} \mathrm{rad} / \mathrm{s}$ for van der Waals pressure $(\alpha=m)$. For $\omega \geqslant 6 \times 10^{16} \mathrm{rad} / \mathrm{s}$, the choice of numerical integration over $q_{\rho}$ or the stationary phase method is based on the magnitude of $k_{0} R$. For the computation of van der Waals pressure along the real frequency axis, the spacing, $\Delta \omega$, between successive frequencies at which the integrand is evaluated is given by $\Delta \omega=\pi c / M d$, where $M$ is the number of frequencies per oscillation period of $2 \pi$ and is set to be 10. The results are shown in Fig. 4. The relative error of calculating the van der Waals pressure between evaluation of Eq. (3) along the real frequency axis and Lifshitz theory calculation along the imaginary axis is $\approx 0.8 \%$. The relative error between the method of surface decomposition and evaluation of Eq. (2) (for energy) or Eq. (3) (for pressure) are $\approx 0.2 \%$ and $\approx 1.1 \%$ for energy flux and van der Waals pressure between two half-spaces, respectively.

\section{ZONES OF INFLUENCE FOR ENERGY TRANSFER AND VAN DER WAALS PRESSURE}

Having ascertained the accuracy of our computation of $S_{1 \rightarrow 2}^{(e)}(\tilde{\mathbf{r}}, \mathbf{r}, \omega)$ and $S_{1 \rightarrow 2}^{(m)}(\tilde{\mathbf{r}}, \mathbf{r}, \omega)$ (by two measures), we now determine the contribution to energy flux and van der Waals pressure at $\tilde{\mathbf{r}}$ from a disk of radius $\rho$ [see Fig. 5(a)]. Keep in mind that the coordinate of $\tilde{\mathbf{r}}$ is $(0,0, d)$. We should stress that the interaction from the disk of radius $\rho$ comes from thermal sources distributed all over half-space 1. Because of axisymmetry, the contributions to energy and momentum transfer from all points within a circular ring of radius $\rho$ and

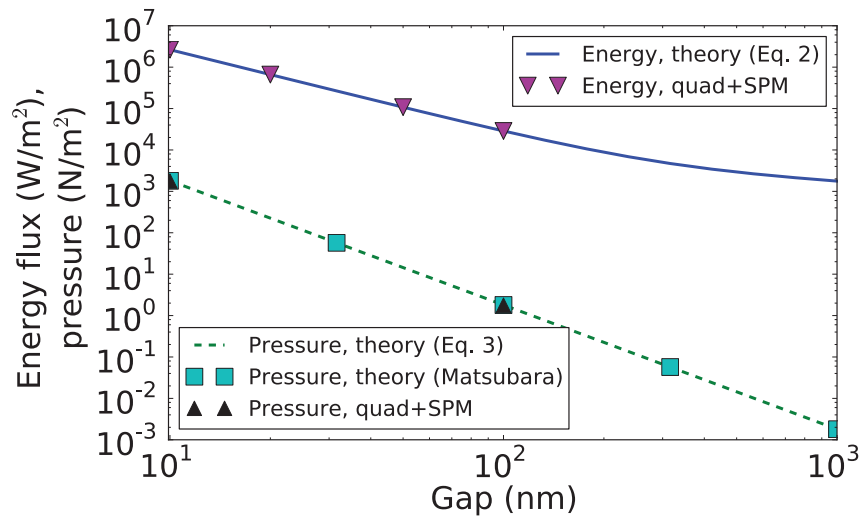

FIG. 4. (Color online) Variation of energy flux (continuous line, evaluating integral in Eq. (2); downward triangle marker, integration of surface patch contributions) and van der Waals pressure (dashed line, evaluating integral in Eq. (3); square marker, calculation according to Lifshitz method using Matsubara frequencies; upward triangle marker, integration of surface patch contributions) between two half-spaces.

thickness $d \rho$ are identical. Instead of plotting $S_{1 \rightarrow 2}^{(\alpha)}(\tilde{\mathbf{r}}, \mathbf{r}, \omega)$ $(\alpha=e, m)$, we plot the fraction of total energy flux (or van der Waals pressure) from a disk of radius $\rho$ on the surface of half-space 1 , i.e.,

$$
\begin{aligned}
p^{(e)}(\rho, d) & =\frac{\int_{0}^{\infty} d \omega \Theta\left(\omega, T_{1}\right) \int_{0}^{\rho} d \rho^{\prime} \rho^{\prime} S_{1 \rightarrow 2}^{(e)}(\tilde{\mathbf{r}}, \mathbf{r}, \omega)}{\int_{0}^{\infty} d \omega \Theta\left(\omega, T_{1}\right) \int_{0}^{\infty} d \rho^{\prime} \rho^{\prime} S_{1 \rightarrow 2}^{(e)}(\tilde{\mathbf{r}}, \mathbf{r}, \omega)} \\
p^{(m)}(\rho, d) & =\frac{\int_{0}^{\infty} d \omega \omega \Theta\left(\omega, T_{1}\right) \int_{0}^{\rho} d \rho^{\prime} \rho^{\prime} S_{1 \rightarrow 2}^{(m)}(\tilde{\mathbf{r}}, \mathbf{r}, \omega)}{\int_{0}^{\infty} d \omega \omega \Theta\left(\omega, T_{1}\right) \int_{0}^{\infty} d \rho^{\prime} \rho^{\prime} S_{1 \rightarrow 2}^{(m)}(\tilde{\mathbf{r}}, \mathbf{r}, \omega)} .
\end{aligned}
$$

In Figs. 5(b)-5(d), we plot $p^{(e)}(\rho, d)$ and $p^{(m)}(\rho, d)$ as a function of $\rho / d$ for $d=10 \mathrm{~nm}$ and $d=100 \mathrm{~nm}$ between two half-spaces with silica, silicon carbide and gold, respectively. The two curves show differences which reflect the differences between energy transfer and van der Waals pressure. Since energy transfer always takes place from higher to lower temperatures, all portions of $S_{1}$ contribute positively to the total energy transfer [as the curves with triangle markers show in Figs. 5(b)-5(d)]. Hence, $p^{(e)}(\rho, d)$ is a monotonically increasing function of $\rho$. Clearly, momentum transfer has no such restrictions and one part of $S_{1}$ contributes an attractive pressure at $\tilde{\mathbf{r}}$ while the rest contributes a repulsive pressure [31,32], even though the total van der Waals pressure is attractive. There are three findings: (1) it can be seen clearly that some portions of the surface contribute to a repulsive pressure-the contributions of the region $\rho \gtrsim 3 d$ of $S_{1}$ for silica [as the curves with circular and square markers show in Fig. 5(b)], the region $\rho \gtrsim 2 d$ for silicon carbide [Fig. 5(c)], and the region $\rho \gtrsim 5 d$ for gold [Fig. 5(d)], to van der Waals pressure are repulsive; (2) to make up for the repulsive contributions from certain regions, van der Waals pressure from other parts of the surface has to be greater than the total pressure between two half-spaces; and (3) for the materials studied here, the extent of the surface required to reach $90 \%$ (or any value close to $100 \%$ ) of total pressure is less than that required to capture the same fraction of total energy transfer. Since the dependence of radiative energy transfer 
(a)

(b)
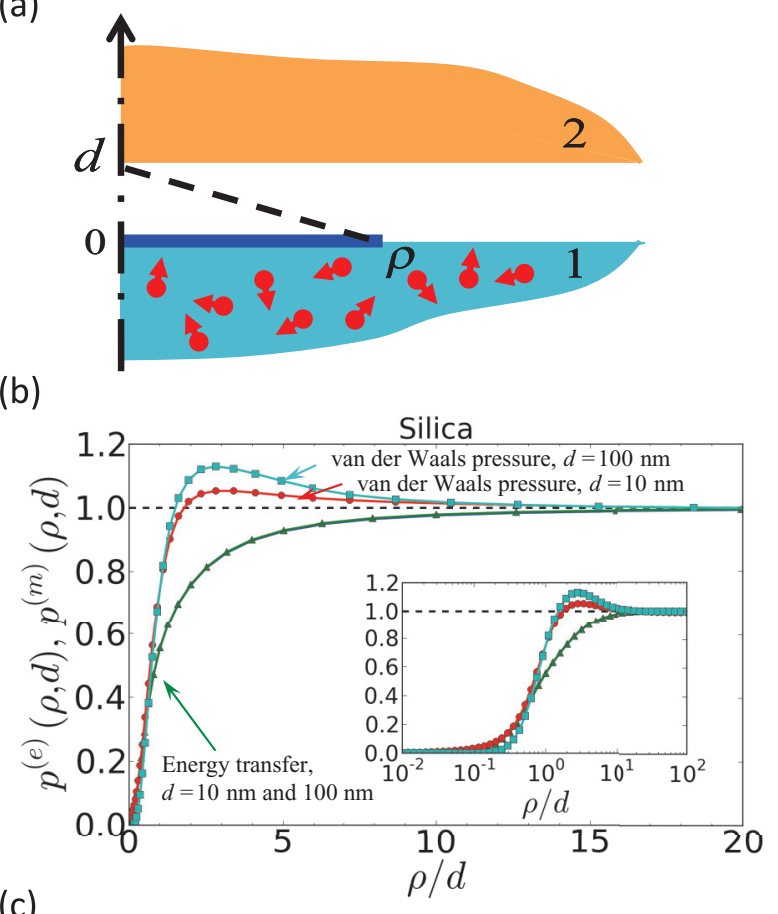

(c)

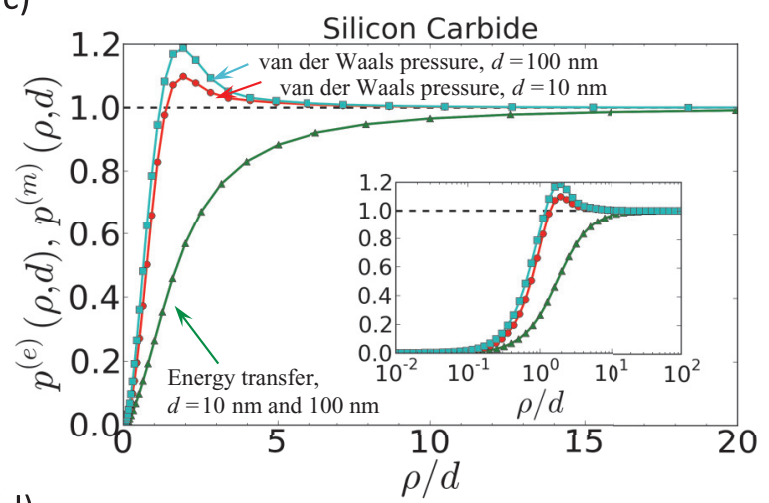

(d)

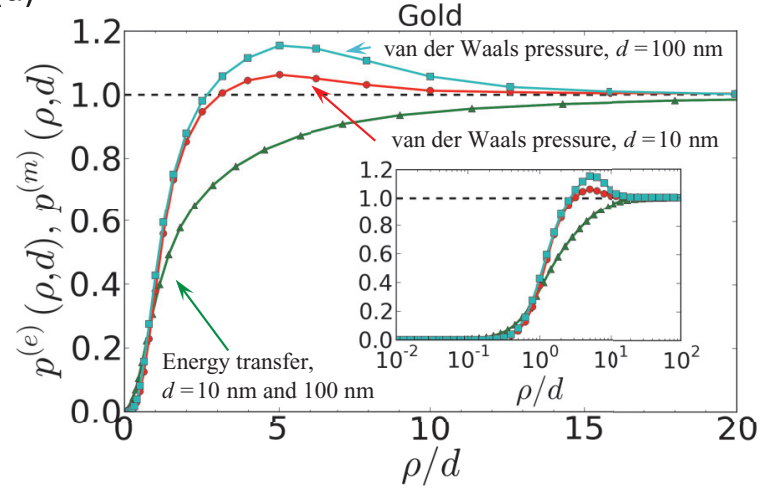

FIG. 5. (Color online) Cumulative partial contributions to energy and momentum transfer from a disk of radius $\rho$ from 0 to $100 d$. (a) Axisymmetric cross section of two half-planes with a circular disk of radius $\rho$ highlighted as the thicker portion of surface $S_{1}$. Thermal sources are distributed throughout the bottom half-space. (b) Silica: cumulative contribution curves as a function of nondimensional fraction $\rho / d$ to radiative energy (with triangle markers) and momentum transfer (with circular or square markers) between two half-spaces. $d=10$ and $100 \mathrm{~nm}$. In the inset, the same curves are shown on a logarithmic $x$ scale. (c) Silicon carbide. (d) Gold. or van der Waals pressure between half-spaces is taken to be the basic result from which the rate of energy transfer or pressure between two curved surfaces is computed by the proximity approximation [33-36], the results presented here seem to indicate that a proximity-like approximation might be more valid for van der Waals and/or Casimir pressure than for radiative energy transfer.

It is worth noting that the cumulative partial contributions to radiative energy and momentum transfer as shown in Fig. 5 are qualitatively similar for both dielectric materials (silica and silicon carbide) and metals (gold), though their optical properties can be significantly different [17]. This is not to say that the magnitudes are similar. In particular, the radiative energy transfer at any gap between silica and silicon carbide half-spaces exceeds that between gold half-spaces considerably [26,28]. It is well known that the surface phonon polaritons, which are excited at 0.061 and $0.142 \mathrm{eV}$ for silica and at $0.117 \mathrm{eV}$ for silicon carbide, can enhance the near-field radiative transfer [37,38], in-plane thermal conductivity of thin films [39], and the optical force in nanostructures [40]. Unlike silica or silicon carbide, a half-space of a metal such as gold is a near-perfect reflector in the visible and near-infrared spectra. Since the plasmon polariton frequencies for metals (e.g., plasma frequency $\omega_{p}=9.019 \mathrm{eV}$ for gold [41]) are higher, the coupling of surface plasmon polaritons at the goldvacuum or gold-dielectric interface contributes significantly to van der Waals pressure $[42,43]$. A systematic investigation of the dependence of $p^{(e)}(\rho, d)$ and $p^{(m)}(\rho, d)$ on the optical properties of the materials will reveal more information but is not pursued in this paper.

We also notice that $p^{(e)}(\rho, d)$ for $d=10 \mathrm{~nm}$ and $d=$ $100 \mathrm{~nm}$ are coincident, whereas they are not for $p^{(m)}(\rho, d)$. This is because contributions to energy transfer arise from evanescent waves at low enough frequencies such that the electrostatic approximation $(\omega d / c \ll 1)$ is valid. In the electrostatic approximation, it can be shown that $p^{(e)}(\rho, d)$ is simply a function of $\rho / d$. This is not so for van der Waals pressure since most of the contributions arise from frequencies where the phase is important.

\section{CONCLUSION}

To summarize, we have calculated the contribution to the energy flux or van der Waals pressure at any location on the surface of one half-space attributable to different portions of the surface of the other half-space. To do so, we have used fluctuational electrodynamics and the dyadic Green's function formalism. Aside from integration along the real frequency axis, our analysis of van der Waals pressure contribution stands apart from most others in literature because of the evaluation of contribution from different parts of the surface of a half-space to the pressure in the vacuum gap via surface decomposition.

We have shown that near-field radiative energy and momentum transfer have different zones of influence of interactions, which could be important to take into consideration when deriving proofs for the applicability of the proximity or modified proximity approximation for fluctuational momentum and energy transfer between curved surfaces $[35,36,44]$. Since certain portions of the surface contribute to a repulsive pressure, which is confirmed for some materials (silica, silicon 
carbide, and gold) in this paper, it may be possible to create objects with net repulsive van der Waals pressure by truncating or texturing the surfaces appropriately [45-48].

\section{ACKNOWLEDGMENT}

This work is funded partially by ONR Grant No. N0001412-1-0996.
[1] S. Rytov, Air Force Cambridge Research Center Technical Report No. AFCRC-TR-59-162, 1967 (unpublished).

[2] L. Landau and E. Lifshitz, Statistical Physics (Pergamon Press, Oxford, 1980), Vol. 24.

[3] M. Antezza, L. P. Pitaevskii, and S. Stringari, Phys. Rev. Lett. 95, 113202 (2005).

[4] L. P. Pitaevskii, Phys. Rev. A 73, 047801 (2006).

[5] Y. Zheng and A. Narayanaswamy, Phys. Rev. A 83, 042504 (2011).

[6] M. Krüger, T. Emig, and M. Kardar, Phys. Rev. Lett. 106, 210404 (2011).

[7] S. Basu and Z. Zhang, J. Appl. Phys. 105, 093535 (2009).

[8] A. Narayanaswamy and Y. Zheng, Phys. Rev. B 88, 075412 (2013).

[9] L. Hu, A. Narayanaswamy, X. Chen, and G. Chen, Appl. Phys. Lett. 92, 133106 (2008).

[10] S.-A. Biehs, E. Rousseau, and J.-J. Greffet, Phys. Rev. Lett. 105, 234301 (2010).

[11] A. Narayanaswamy and Y. Zheng, J. Quant. Spectrosc. Radiat. Transfer 132, 12 (2014).

[12] W. C. Chew, Waves and Fields in Inhomogeneous Media (IEEE, New York, 1995).

[13] V. A. Parsegian, Van der Waals Forces: A Handbook for Biologists, Chemists, Engineers, and Physicists (Cambridge University Press, Cambridge, UK, 2006).

[14] A. Narayanaswamy and Y. Zheng, Phys. Rev. A 88, 012502 (2013).

[15] E. M. Lifshitz, Sov. Phys. JETP 2, 73 (1956).

[16] R. S. Ottens, V. Quetschke, S. Wise, A. A. Alemi, R. Lundock, G. Mueller, D. H. Reitze, D. B. Tanner, and B. F. Whiting, Phys. Rev. Lett. 107, 014301 (2011).

[17] E. D. Palik, Handbook of Optical Constants of Solids (Academic, New York, 1998), Vol. 3.

[18] L. Mandel and E. Wolf, Optical Coherence and Quantum Optics (Cambridge University Press, Cambridge, UK, 1995).

[19] M. Francoeur and M. Pinar Mengüç, J. Quant. Spectrosc. Radiat. Transfer 109, 280 (2008).

[20] M. Francoeur, M. Pinar Mengüç, and R. Vaillon, J. Quant. Spectrosc. Radiat. Transfer 110, 2002 (2009).

[21] C.-T. Tai, Dyadic Green Functions in Electromagnetic Theory (IEEE, New York, 1994), Vol. 272.

[22] A. Narayanaswamy and G. Chen, J. Quant. Spectrosc. Radiat. Transfer 111, 1877 (2010).

[23] V. A. Parsegian and B. W. Ninham, J. Theor. Biol. 38, 101 (1973).

[24] M. Paulus, P. Gay-Balmaz, and O. J. F. Martin, Phys. Rev. E 62, 5797 (2000).
[25] P. W. Milonni and C. Eberlein, The Quantum Vacuum: An Introduction to Quantum Electrodynamics (Academic, San Diego, 1994), Vol. 1.

[26] S. Basu, Z. Zhang, and C. Fu, Int. J. Energy Res. 33, 1203 (2009).

[27] P. Yla-Oijala and M. Taskinen, IEEE Trans. Antennas Propag. 51, 2106 (2003).

[28] K. Joulain, J.-P. Mulet, F. Marquier, R. Carminati, and J.-J. Greffet, Surf. Sci. Rep. 57, 59 (2005).

[29] C. Eckart, Rev. Mod. Phys. 20, 399 (1948).

[30] M. Antezza, L. P. Pitaevskii, S. Stringari, and V. B. Svetovoy, Phys. Rev. A 77, 022901 (2008).

[31] F. W. DelRio, M. P. de Boer, J. A. Knapp, E. D. Reedy, P. J. Clews, and M. L. Dunn, Nat. Mater. 4, 629 (2005).

[32] J. N. Munday, F. Capasso, and V. A. Parsegian, Nature (London) 457, 170 (2009).

[33] K. Sasihithlu and A. Narayanaswamy, Phys. Rev. B 83, 161406 (2011).

[34] H. Gies and K. Klingmüller, Phys. Rev. Lett. 96, 220401 (2006).

[35] M. Krüger, V. A. Golyk, G. Bimonte, and M. Kardar, Europhys. Lett. 104, 41001 (2013).

[36] C. D. Fosco, F. C. Lombardo, and F. D. Mazzitelli, Phys. Rev. D 84, 105031 (2011).

[37] M. Francoeur, S. Basu, and S. J. Petersen, Opt. Express 19, 18774 (2011).

[38] S. Shen, A. Narayanaswamy, and G. Chen, Nano Lett. 9, 2909 (2009).

[39] Dye-Zone A. Chen, A. Narayanaswamy, and G. Chen, Phys. Rev. B 72, 155435 (2005).

[40] D. Li, N. M. Lawandy, and R. Zia, Opt. Express 21, 20900 (2013).

[41] R. L. Olmon, B. Slovick, T. W. Johnson, D. Shelton, S.-H. Oh, G. D. Boreman, and M. B. Raschke, Phys. Rev. B 86, 235147 (2012).

[42] C. Henkel, K. Joulain, J.-P. Mulet, and J.-J. Greffet, Phys. Rev. A 69, 023808 (2004).

[43] S. T. Koev, A. Agrawal, H. J. Lezec, and V. A. Aksyuk, Plasmonics 7, 269 (2012).

[44] V. A. Golyk, M. Krüger, A. P. McCauley, and M. Kardar, Europhys. Lett. 101, 34002 (2013).

[45] M. Levin, A. P. McCauley, A. W. Rodriguez, M. T. Homer Reid, and S. G. Johnson, Phys. Rev. Lett. 105, 090403 (2010).

[46] A. W. Rodriguez, F. Capasso, and S. G. Johnson, Nat. Photonics 5, 211 (2011).

[47] R. Maboudian and R. T. Howe, J. Vac. Sci. Technol. B 15, 1 (1997).

[48] J. N. Israelachvili, Intermolecular and Surface Forces, revised 3rd ed. (Academic, New York, 2011). 